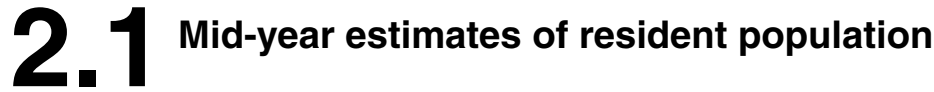

Thousands

\begin{tabular}{|c|c|c|c|c|c|c|c|c|c|c|c|c|}
\hline & & and and W & & & Scotland & & & orthern Ire & & & hited Kingd & \\
\hline & Males & Females & Persons & Males & Females & Persons & Males & Females & Persons & Males & Females & Persons \\
\hline & BBAE & BBAF & BBAD & $\mathrm{BBAH}$ & BBAI & BBAG & BBAK & BBAL & BBAJ & BBAB & BBAC & DYAY \\
\hline 1986 & 24311 & 25687 & 49999 & 2462 & 2649 & 5112 & 768 & 805 & 1574 & 27542 & 29142 & 56684 \\
\hline 1987 & 24371 & 25752 & 50123 & 2455 & 2644 & 5099 & 773 & 809 & 1582 & 27599 & 29205 & 56804 \\
\hline 1988 & 24434 & 25820 & 50254 & 2444 & 2633 & 5077 & 774 & 812 & 1585 & 27652 & 29265 & 56916 \\
\hline 1989 & 24510 & 25898 & 50408 & 2443 & 2635 & 5078 & 776 & 814 & 1590 & 27729 & 29348 & 57076 \\
\hline 1990 & 24597 & 25964 & 50561 & 2444 & 2637 & 5081 & 778 & 818 & 1596 & 27819 & 29419 & 57237 \\
\hline 1991 & 24681 & 26067 & 50748 & 2445 & 2639 & 5083 & 783 & 824 & 1607 & 27909 & 29530 & 57439 \\
\hline 1992 & 24739 & 26136 & 50876 & 2445 & 2640 & 5086 & 792 & 831 & 1623 & 27977 & 29608 & 57585 \\
\hline 1993 & 24793 & 26193 & 50986 & 2448 & 2644 & 5092 & 798 & 837 & 1636 & 28039 & 29675 & 57714 \\
\hline 1994 & 24853 & 26263 & 51116 & 2453 & 2649 & 5102 & 802 & 842 & 1644 & 28108 & 29754 & 57862 \\
\hline 1995 & 24946 & 26326 & 51272 & 2453 & 2650 & 5104 & 804 & 845 & 1649 & 28204 & 29821 & 58025 \\
\hline 1996 & 25030 & 26381 & 51410 & 2447 & 2645 & 5092 & 810 & 851 & 1662 & 28287 & 29877 & 58164 \\
\hline 1997 & 25113 & 26446 & 51560 & 2442 & 2641 & 5083 & 816 & 856 & 1671 & 28371 & 29943 & 58314 \\
\hline 1998 & 25201 & 26519 & 51720 & 2439 & 2638 & 5077 & 819 & 859 & 1678 & 28458 & 30017 & 58475 \\
\hline 2000 & 25438 & 26702 & 52140 & 2432 & 2631 & 5063 & 820 & 862 & 1683 & 28690 & 30196 & 58886 \\
\hline 2001 & 25574 & 26786 & 52360 & 2434 & 2630 & 5064 & 824 & 865 & 1689 & 28832 & 30281 & 59113 \\
\hline 2002 & 25704 & 26868 & 52572 & 2432 & 2623 & 5055 & 829 & 868 & 1697 & 28964 & 30359 & 59323 \\
\hline 2003 & 25841 & 26956 & 52797 & 2435 & 2623 & 5057 & 833 & 870 & 1703 & 29109 & 30449 & 59557 \\
\hline 2004 & 25995 & 27062 & 53057 & 2446 & 2632 & 5078 & 836 & 874 & 1710 & 29278 & 30568 & 59846 \\
\hline 2005 & 26197 & 27223 & 53419 & 2456 & 2639 & 5095 & 844 & 880 & 1724 & 29497 & 30741 & 60238 \\
\hline 2006 & 26371 & 27358 & 53729 & 2469 & 2647 & 5117 & 853 & 888 & 1742 & 29694 & 30893 & 60587 \\
\hline 2007 & 26569 & 27503 & 54072 & 2486 & 2659 & 5144 & 862 & 897 & 1759 & 29916 & 31059 & 60975 \\
\hline
\end{tabular}

\subsection{Age distribution of estimated resident population at 30 June 2007}

Thousands

\begin{tabular}{|c|c|c|c|c|c|c|c|c|c|c|c|}
\hline & \multicolumn{11}{|c|}{ Resident population } \\
\hline & \multicolumn{2}{|c|}{ England and Wales ${ }^{1}$} & \multicolumn{2}{|c|}{ Wales } & \multicolumn{2}{|c|}{ Scotland } & \multicolumn{2}{|c|}{ Northern Ireland } & \multicolumn{3}{|c|}{ United Kingdom ${ }^{1}$} \\
\hline & Males & Females & Males & Females & Males & Females & Males & Females & Males & Females & Persons \\
\hline $0-4$ & 1641 & 1562 & 84 & 80 & 141 & 134 & 59 & 56 & 1841 & 1752 & 3593 \\
\hline $5-9$ & 1552 & 1484 & 85 & 81 & 140 & 134 & 59 & 56 & 1750 & 1674 & 3424 \\
\hline $10-14$ & 1680 & 1598 & 96 & 91 & 155 & 148 & 63 & 60 & 1898 & 1806 & 3704 \\
\hline $15-19$ & 1834 & 1724 & 105 & 99 & 169 & 160 & 66 & 62 & 2069 & 1947 & 4016 \\
\hline $20-24$ & 1881 & 1780 & 104 & 99 & 175 & 172 & 68 & 64 & 2124 & 2016 & 4141 \\
\hline $25-29$ & 1770 & 1756 & 84 & 84 & 164 & 160 & 58 & 58 & 1992 & 1974 & 3966 \\
\hline $30-34$ & 1738 & 1735 & 79 & 82 & 149 & 156 & 57 & 58 & 1944 & 1949 & 3893 \\
\hline $35-39$ & 2003 & 2028 & 97 & 104 & 180 & 195 & 63 & 65 & 2246 & 2288 & 4534 \\
\hline $40-44$ & 2073 & 2105 & 106 & 111 & 195 & 211 & 64 & 67 & 2332 & 2382 & 4714 \\
\hline $45-49$ & 1853 & 1891 & 99 & 104 & 187 & 199 & 59 & 62 & 2099 & 2151 & 4250 \\
\hline $50-54$ & 1622 & 1660 & 92 & 96 & 168 & 175 & 52 & 52 & 1842 & 1888 & 3730 \\
\hline $55-59$ & 1635 & 1686 & 98 & 101 & 162 & 169 & 47 & 49 & 1845 & 1903 & 3748 \\
\hline $60-64$ & 1511 & 1581 & 93 & 96 & 146 & 155 & 44 & 46 & 1701 & 1782 & 3483 \\
\hline $65-69$ & 1148 & 1234 & 72 & 77 & 114 & 130 & 34 & 37 & 1296 & 1401 & 2697 \\
\hline $70-74$ & 979 & 1108 & 60 & 67 & 96 & 118 & 28 & 33 & 1102 & 1259 & 2360 \\
\hline $75-79$ & 769 & 982 & 46 & 58 & 71 & 100 & 20 & 29 & 861 & 1111 & 1972 \\
\hline $80-84$ & 513 & 785 & 32 & 49 & 44 & 74 & 13 & 22 & 570 & 881 & 1452 \\
\hline $85-89$ & 268 & 520 & 16 & 32 & 21 & 45 & 6 & 13 & 295 & 578 & 873 \\
\hline 90 and over & 99 & 286 & 6 & 16 & 8 & 24 & 2 & 6 & 108 & 317 & 425 \\
\hline $0-14$ & 4872 & 4644 & 265 & 251 & 436 & 415 & 182 & 172 & 5490 & 5231 & 10721 \\
\hline $15-64$ & 17921 & 17945 & 957 & 975 & 1696 & 1752 & 577 & 585 & 20194 & 20281 & 40475 \\
\hline 65 and over & 3775 & 4915 & 231 & 299 & 354 & 491 & 103 & 140 & 4233 & 5546 & 9779 \\
\hline All ages & 26569 & 27503 & 1454 & 1526 & 2486 & 2659 & 862 & 897 & 29916 & 31059 & 60975 \\
\hline
\end{tabular}




\section{Births $^{1}$ and marriages}

Thousands

\begin{tabular}{|c|c|c|c|c|c|c|c|c|c|c|c|c|c|}
\hline & \multicolumn{6}{|c|}{ Live births ${ }^{2,3}$} & \multicolumn{6}{|c|}{ Marriages } & \multirow{3}{*}{$\begin{array}{l}\text { Civil } \\
\text { Partnersh- } \\
\text { ips }^{4} \\
\\
\text { United } \\
\text { Kingdom }\end{array}$} \\
\hline & \multicolumn{3}{|c|}{ England and Wales } & \multirow[b]{2}{*}{ Scotland } & \multirow{2}{*}{$\begin{array}{l}\text { Northern } \\
\text { Ireland }\end{array}$} & \multirow{2}{*}{$\begin{array}{r}\text { United } \\
\text { Kingdom }\end{array}$} & \multicolumn{3}{|c|}{ England and Wales } & \multirow[b]{2}{*}{ Scotland } & \multirow{2}{*}{$\begin{array}{l}\text { Northern } \\
\text { Ireland }\end{array}$} & \multirow{2}{*}{$\begin{array}{r}\text { United } \\
\text { Kingdom }\end{array}$} & \\
\hline & Total & England & Wales & & & & Total & England & Wales & & & & \\
\hline & BBCB & G8ZT & BBCC & BBCD & BBCE & BBCA & BBCG & G8ZU & $\mathrm{BBCH}$ & $\mathrm{BBCl}$ & BBCJ & BBCF & HF79 \\
\hline 2004 & 639.7 & 607.2 & 32.3 & 54.0 & 22.3 & 716.0 & 273.1 & 258.2 & 14.9 & 32.2 & 8.3 & 313.6 & .. \\
\hline 2006 & 669.6 & 635.7 & 33.6 & 55.7 & 23.3 & 748.6 & 239.5 & 226.0 & 13.5 & 29.9 & 8.3 & 277.6 & 16.1 \\
\hline 2007 & 690.0 & 655.4 & 34.4 & 57.8 & 24.5 & 772.2 & $231.4^{5}$ & $215.6^{5}$ & $12.8^{5}$ & 29.9 & 8.7 & $270.0^{5}$ & $8.7^{5}$ \\
\hline 2008 & .. & .. & .. & .. & .. & .. &.. &.. & .. & $28.9^{5}$ & .. & .. & .. \\
\hline 2005 Q2 & 159.8 & 151.8 & 7.9 & 13.6 & 5.7 & 179.0 & 68.2 & 64.3 & 3.9 & 8.6 & 2.2 & 79.0 & .. \\
\hline Q3 & 170.2 & 161.4 & 8.7 & 14.2 & 5.9 & 190.3 & 105.3 & 99.3 & 6.0 & 12.3 & 3.5 & 121.1 & .. \\
\hline Q4 & 161.7 & 153.4 & 8.2 & 13.2 & 5.2 & 180.1 & 44.0 & 41.4 & 2.5 & 6.1 & 1.4 & 51.5 & 2.0 \\
\hline 2006 Q1 & 159.5 & 151.4 & 8.1 & 13.6 & 5.8 & 178.9 & 25.9 & 24.4 & 1.4 & 3.5 & 0.9 & 30.3 & 4.9 \\
\hline Q4 & 169.0 & 160.5 & 8.4 & 13.9 & 5.6 & 188.5 & 41.2 & 38.9 & 2.2 & 5.9 & 1.5 & 48.6 & 2.4 \\
\hline 2007 Q1 & 164.0 & 155.9 & 8.1 & 14.2 & 6.1 & 184.4 & $26.7^{5}$ & $25.4^{5}$ & $1.3^{5}$ & 3.3 & 1.0 & $31.0^{5}$ & 1.7 \\
\hline Q2 & 169.5 & 161.0 & 8.5 & 14.3 & 6.0 & 189.8 & $65.2^{5}$ & $61.6^{5}$ & $3.6^{5}$ & 8.1 & 2.4 & $75.7^{5}$ & 2.4 \\
\hline Q3 & 181.4 & 172.2 & 9.1 & 14.9 & 6.5 & 202.8 & $99.4^{5}$ & $93.7^{5}$ & $5.7^{5}$ & 12.6 & 3.8 & $115.8^{5}$ & 3.0 \\
\hline Q4 & 175.0 & 166.3 & 8.7 & 14.4 & 5.9 & 195.3 & $40.1^{5}$ & $37.9^{5}$ & $2.2^{5}$ & 5.8 & 1.6 & $47.4^{5}$ & 1.7 \\
\hline 2008 Q1 & $173.8^{5}$ & $165.0^{5}$ & $8.7^{5}$ & $15.1^{5}$ & $6.5^{5}$ & $195.4^{5}$ & .. & .. & .. & $3.4^{5}$ & $1.1^{5}$ & .. & $1.3^{5}$ \\
\hline Q2 & $176.8^{5}$ & $167.8^{5}$ & $9.0^{5}$ & $14.9^{5}$ & $6.3^{5}$ & $198.0^{5}$ & .. & .. & .. & $7.9^{5}$ & $2.2^{5}$ & .. & $1.9^{5}$ \\
\hline Q3 & $182.0^{5}$ & $172.8^{5}$ & $9.2^{5}$ & $15.5^{5}$ & $6.5^{5}$ & $204.0^{5}$ & .. & .. & .. & $11.9^{5}$ & $3.6^{5}$ & .. & $2.5^{5}$ \\
\hline Q4 & 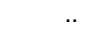 & 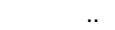 &.. &.. &.. &.. & .. & .. & .. & $5.7^{5}$ &.. & .. & .. \\
\hline
\end{tabular}

Note: Figures may not add exactly due to rounding.

1 Excluding stillbirths.

2 Birth figures for England and also for Wales each exclude events

for persons usually resident outside England and Wales. These events

are however, included in the totals for England and Wales combined, and

for the United Kingdom.
3 For England and Wales, figures relate to numbers occurring in a period; for Scotland and Northern Ireland, figures relate to those registered in a period.

4 The Civil Partnership Act 2004 came into force on 5 December 2005 in the UK 5 Provisional

Sources: Office for National Statistics (Population Trends No 135) General Register Office for Scotland; Northern Ireland Statistics \& Research Agency.

\subsection{Deaths registered}

Thousands

\begin{tabular}{|c|c|c|c|c|c|c|c|c|c|c|}
\hline & \multicolumn{5}{|c|}{ Total } & \multicolumn{5}{|c|}{ Infants aged under one year } \\
\hline & \multicolumn{2}{|c|}{ England and Wales } & \multirow[b]{2}{*}{ Scotland } & \multirow{2}{*}{$\begin{array}{l}\text { Northern } \\
\text { Ireland }\end{array}$} & \multirow{2}{*}{$\begin{array}{r}\text { United } \\
\text { Kingdom }\end{array}$} & \multicolumn{2}{|c|}{ England and Wales } & \multirow[b]{2}{*}{ Scotland } & \multirow{2}{*}{$\begin{array}{l}\text { Northern } \\
\text { Ireland }\end{array}$} & \multirow{2}{*}{$\begin{array}{r}\text { United } \\
\text { Kingdom }\end{array}$} \\
\hline & Total & Wales & & & & Total & Wales & & & \\
\hline & BBDB & BBDC & BBDD & BBDE & BBDA & BBDG & $\mathrm{BBDH}$ & BBDI & BBDJ & BBDF \\
\hline 2003 & 539.2 & 33.8 & 58.4 & 14.5 & 612.0 & 3.30 & 0.13 & 0.27 & 0.12 & 3.69 \\
\hline 2004 & 514.3 & 32.3 & 56.2 & 14.4 & 584.8 & 3.27 & 0.16 & 0.27 & 0.12 & 3.66 \\
\hline 2005 & 513.0 & 32.2 & 55.7 & 14.2 & 583.0 & 3.25 & 0.14 & 0.28 & 0.14 & 3.67 \\
\hline 2007 & 504.1 & 32.1 & 56.1 & 14.6 & 575.1 & 3.35 & 0.18 & 0.27 & 0.12 & 3.74 \\
\hline 2005 Q1 & 145.3 & 9.2 & 15.6 & 3.8 & 164.7 & 0.82 & 0.03 & 0.07 & 0.03 & 0.91 \\
\hline Q2 & 125.9 & 8.0 & 13.7 & 3.7 & 143.3 & 0.83 & 0.04 & 0.07 & 0.04 & 0.94 \\
\hline Q3 & 115.4 & 7.2 & 12.8 & 3.4 & 131.6 & 0.80 & 0.03 & 0.08 & 0.04 & 0.92 \\
\hline Q4 & 126.2 & 7.8 & 13.6 & 3.4 & 143.3 & 0.80 & 0.04 & 0.07 & 0.03 & 0.90 \\
\hline 2006 Q1 & 141.0 & 8.7 & 14.9 & 4.0 & 159.9 & 0.82 & 0.03 & 0.05 & 0.03 & 0.90 \\
\hline Q2 & 123.9 & 7.6 & 13.9 & 3.6 & 141.4 & 0.84 & 0.03 & 0.07 & 0.03 & 0.94 \\
\hline 2007 Q1 & 139.3 & 8.8 & 15.8 & 4.2 & 159.3 & 0.80 & 0.05 & 0.07 & 0.04 & 0.91 \\
\hline Q2 & 121.0 & 7.9 & 13.4 & 3.6 & 138.0 & 0.88 & 0.04 & 0.08 & 0.03 & 0.99 \\
\hline Q3 & 114.0 & 7.3 & 12.6 & 3.3 & 129.9 & 0.84 & 0.04 & 0.07 & 0.03 & 0.94 \\
\hline Q4 & 129.7 & 8.3 & 14.2 & 3.6 & 147.5 & 0.83 & 0.05 & 0.06 & 0.02 & 0.90 \\
\hline $2008 \mathrm{Q} 1^{1}$ & 136.1 & 8.6 & 15.1 & 4.1 & 155.4 & 0.86 & 0.04 & 0.06 & 0.03 & 0.95 \\
\hline Q2 1 & 122.7 & 7.7 & 13.5 & 3.6 & 139.8 & 0.79 & 0.04 & 0.06 & 0.03 & 0.88 \\
\hline $\mathrm{Q}^{1}$ & 113.8 & 7.3 & 12.6 & 3.4 & 129.8 & 0.86 & 0.04 & 0.07 & 0.03 & 0.96 \\
\hline
\end{tabular}

